\title{
A note on simultaneous polynomial approximation of exponential functions
}

\section{J.H. Loxton and A.J. van der Poorten}

Let $\alpha_{1}, \ldots, \alpha_{m}$ be distinct complex numbers and $\tau(1), \ldots, \tau(m)$ be non-negative integers. We obtain conditions under which the functions

$$
z^{\tau(1)} \exp \left(\alpha_{1} z\right), \ldots, z^{\tau(m)} \exp \left(\alpha_{m} z\right)
$$

form a perfect system, that is, for every set $\rho(1), \ldots, \rho(m)$ of non-negative integers, there are polynomials $a_{1}(z), \ldots, a_{m}(z)$, with respective degrees exactly $\rho(1)-1, \ldots, \rho(m)-1$, such that the function

$$
R(z)=\sum_{k=1}^{m} a_{k}(z) \exp \left(\alpha_{k} z\right)
$$

has a zero of order at least $\rho(1)+\ldots+\rho(m)-1$ at the origin. Moreover, subject to the evaluation of certain determinants, we give explicit formulae for the approximating polynomials $a_{1}(z), \ldots, a_{m}(z)$.

\section{Introduction}

In [4], Mahler has introduced the idea of a perfect system of functions, defined as follows. Let $f_{1}(z), \ldots, f_{m}(z)$ be functions of one complex variable which are regular at the origin and do not all vanish there. Let $\rho=(\rho(1), \ldots, \rho(m))$ be an m-tuple of non-negative integers

Received 1 July 1974. 
and set $\sigma=\rho(1)+\ldots+\rho(m)$. Then there are polynomials

$a_{1}(z), \ldots, a_{m}(z)$, with respective degrees at most $\rho(1)-1, \ldots, \rho(m)-1$ and not all identically zero, such that the function

$$
R(z)=\sum_{k=1}^{m} a_{k}(z) f_{k}(z)
$$

has a zero of order at least $\sigma-1$ at the origin. The functions $f_{1}(z), \ldots, f_{m}(z)$ form a perfect system if, for every choice of $\rho$, there are polynomials $a_{1}(z), \ldots, a_{m}(z)$ with respective degrees exactly $\rho(1)-1, \ldots, \rho(m)-1$ such that the function $R(z)$ defined in (1) has a zero of order at least $\sigma-1$ at the origin. The polynomials $a_{1}(z), \ldots, a_{m}(z)$ are then uniquely determined up to a common constant multiple; (see [4], page 113).

In [5], the second author gave several examples of sets of functions whose perfectness can be established by explicitly constructing the approximating polynomials $a_{1}(z), \ldots, a_{m}(z)$. In this note, we consider in the same spirit the perfectness of the system of functions

$$
z^{\tau(1)} \exp \left(\alpha_{1} z\right), \ldots, z^{\tau(m)} \exp \left(\alpha_{m} z\right)
$$

where $\alpha_{1}, \ldots, \alpha_{m}$ are complex numbers and $\tau(1), \ldots, \tau(m)$ are nonnegative integers. The main result and the corresponding construction are given in Section 2.

The particular case $\tau(1)=\ldots=\tau(m)=0$ gives the approximating polynomials constructed by Mahler [2,3] and used by him to obtain arithmetic properties of the exponential function. A lemma on the simultaneous polynomial approximation of the general system (2) was used recently by Baker [1] in obtaining a new diophantine inequality involving the exponential function. Unfortunately, our construction, at least in its present form, does not appear to have any applications of this kind.

\section{Construction of the approximating polynomials}

Let $\alpha=\left(\alpha_{1}, \ldots, \alpha_{m}\right)$ be an m-tuple of complex numbers and $\rho=(\rho(1), \ldots, \rho(m))$ and $\tau=(\tau(1), \ldots, \tau(m))$ be m-tuples of non- 
negative integers. Set $\sigma=\rho(1)+\ldots+\rho(m)$. We denote by $D(\alpha, \rho, \tau)$ the determinant of order $\sigma$ with the element

$$
\left(\begin{array}{c}
i-1 \\
\tau(r)+s-1
\end{array}\right) \alpha_{r}^{i-\tau(r)-s}
$$

in the $i$ th row and $j$ th column, where $j=\rho(1)+\ldots+\rho(r-1)+s$ $(1 \leq r \leq m, 1 \leq s \leq \rho(r))$.

THEOREM. Let $\alpha_{1}, \ldots, \alpha_{m}$ be distinct complex numbers and $\tau(1), \ldots, \tau(m)$ be non-negative integers with

$$
0=\tau(1) \leq \tau(2) \leq \ldots \leq \tau(m) \text {. }
$$

If, for each m-tuple $\rho=(\rho(1), \ldots, \rho(m))$ of non-negative integers, the determinant $D(\alpha, \rho, \tau)$ defined above is non-zero, then the functions

$$
z^{\tau(1)} \exp \left(\alpha_{1} z\right), \ldots, z^{\tau(m)} \exp \left(\alpha_{m} z\right)
$$

form a perfect system.

Proof. Let $\rho=(\rho(1), \ldots, \rho(m))$ be an m-tuple of non-negative integers and set $\sigma=\rho(1)+\ldots+\rho(m)$. Let $\omega_{r s}(1 \leq r \leq m$, $1 \leq \varepsilon \leq \rho(r)$ ) be $\sigma$ distinct complex numbers and denote their difference product by $\Delta(\omega)$. Thus

$$
\Delta(\omega)=\Delta\left(\omega_{11}, \ldots, \omega_{m, \rho(m)}\right)=\left|\omega_{r s}^{i-1}\right|
$$

is the determinant of order $\sigma$ with $\omega_{r s}^{i-l}$ in the $i$ th row and $j$ th column, where $j=\rho(1)+\ldots+\rho(r-1)+s \quad(1 \leq r \leq m, I \leq s \leq \rho(r))$.

Define a function $S(z)$ by

$$
S(z)=\frac{\Delta(\omega)}{2 \pi i} \int_{C} \prod_{r, s}\left(\zeta-\omega_{r s}\right)^{-1} e^{\zeta z} d \zeta,
$$

where $C$ is a simple closed contour in the $\zeta$-plane containing all the $\omega_{r s}$. On the one hand, evaluating the integral by obtaining the residue of the integrand at each of the poles $\omega_{r s}$ inside $C$, we obtain

$$
S(z)=\sum_{r, s} \Delta_{r s}(\omega) \exp \left(\omega_{r s} z\right)
$$


where

$$
\Delta_{r s}(\omega)=(-1)^{\sigma(r, s)} \Delta\left(\omega_{11}, \ldots, \hat{\omega}_{r s}, \ldots, \omega_{m, \rho(m)}\right)
$$

is, except for sign, the difference product of the $\omega_{k l}$ with $\omega_{r s}$ omitted, and we have introduced $\sigma(r, s)=\rho(1)+\ldots+\rho(p-1)+s-1$. In particular, $\Delta_{r s}(\omega)$ is independent of $\omega_{r s}$. On the other hand, evaluating the integral (4) by considering the behaviour of the integrand at its remaining singularity at $\zeta=\infty$, we see that $S(z)$ has a Taylor expansion about the origin which begins

$$
S(z)=\Delta(\omega) \frac{z^{\sigma-1}}{(\sigma-1) !}+\ldots
$$

Define the differential operators

$$
L=\left.\prod_{k, Z} \frac{1}{(\tau(k)+l-1) !}\left(\frac{\partial}{\partial \omega_{k l}}\right)^{\tau(k)+l-1}\right|_{\omega_{k l}=\alpha_{k}},
$$

and, for each pair $(r, s)$ with $1 \leq r \leq m, 1 \leq \varepsilon \leq \rho(r)$,

$$
L_{r s}=\left.\prod_{(k, Z) \neq(r, \delta)} \frac{1}{(\tau(k)+\tau-1) !}\left(\frac{\partial}{\partial \omega_{k l}}\right)^{\tau(k)+z-1}\right|_{\omega_{k l}=\alpha_{k}},
$$

where, after differentiation, we replace each $\omega_{k l}$ by $\alpha_{k}$. On applying. the operator $L$ to (5), we obtain

$$
R(z)=\operatorname{LS}(z)=\sum_{k=1}^{m} a_{k}(z) z^{\tau(k)} \exp \left(\alpha_{k} z\right),
$$

where

$$
a_{k}(z)=\sum_{l=1}^{\rho(k)} \frac{1}{(\tau(k)+l-1) !} L_{k l} \Delta_{k l}(\omega) z^{l-1} \quad(1 \leq k \leq m)
$$

is a polynomial of degree at most $\rho(k)-1$ in $z$. From (6), the function $R(z)$ has a zero of order at least $\sigma-1$ at the origin and its Taylor expansion about the origin begins

$$
R(z)=L \Delta(\omega) \frac{z^{\sigma-1}}{(\sigma-1) !}+\ldots=D(\alpha, \rho, \tau) \frac{z^{\sigma-1}}{(\sigma-1) !}+\ldots
$$


Moreover, the leading coefficient of the polynomial $a_{k}(z)$ is

$$
\pm \frac{1}{(\tau(k)+\rho(k)-1) !} D\left(\alpha, \rho_{k}^{\prime}, \tau\right) \text {, }
$$

where $\rho_{k}^{\prime}=(\rho(1), \ldots, \rho(k)-1, \ldots, \rho(m))$, so by hypothesis, $a_{k}(z)$ has exact degree $\rho(k)-1$. Thus the functions (3) form a perfect system.

\section{Construction of linearly independent approximations}

Following the general theory of [4], pages 104-107, we can use the preceding work to construct explicitly systems of linearly independent forms in the functions (3).

As before, let $\alpha_{1}, \ldots, \alpha_{m}$ be distinct complex numbers and $\tau(1), \ldots, \tau(m)$ be non-negative integers satisfying the hypotheses of the theorem of Section 2. We carry out the construction of Section 2 with the parameters $\rho$ replaced in turn by the m-tuple

$$
\rho_{h}=(\rho(1), \ldots, \rho(h)+1, \ldots, \rho(m)) \quad(1 \leq h \leq m) \text {, }
$$

denoting quantities obtained from $\rho_{h}$ by a subscript $h$. Thus, from (7) and (9), we obtain the functions

$$
\begin{aligned}
R_{h}(z) & =\sum_{k=1}^{m} a_{h k}(z) z^{\tau(k)} \exp \left(\alpha_{k} z\right) \\
& =D\left(\alpha, \rho_{h}, \tau\right) \frac{z^{\sigma}}{\sigma !}+\ldots \quad(1 \leq h \leq m),
\end{aligned}
$$

where, by (8) and (10), $a_{h k}(z)$ is a polynomial in $z$ of degree $\rho(k)+\delta_{h k}$ and the leading coefficient of $a_{k k}(z)$ is

$$
\pm \frac{D(\alpha, \rho, \tau)}{(\tau(k)+\rho(k)) !}
$$

Let $A(z)$ be the $m \times m$ determinant

$$
A(z)=\left|a_{h k}(z)\right|_{1 \leq h, k \leq m} \text {. }
$$

From (11) and the hypothesis $\tau(1)=0$, it follows that $A(z)$ has a zero of order at least $\sigma$ at the origin. On the other hand, from the above remarks, $A(z)$ is a polynomial of degree at most $\sigma$ and, in the expansion 
of $A(z)$, a term of degree $\sigma$ can only arise from the main diagonal. Using (12) to compute this term, we find

$$
A(z)= \pm\left\{\prod_{k=1}^{m} \frac{D(\alpha, \rho, \tau)}{(\tau(k)+\rho(k)) !}\right\} z^{\sigma} .
$$

In particular, from our hypothesis, $A(1) \neq 0$, so on writing $z=1$ in (11), we obtain $m$ linearly independent forms in $\exp \left(\alpha_{1}\right), \ldots, \exp \left(\alpha_{m}\right)$, say

$$
R_{h}=\sum_{k=1}^{m} a_{h k} \exp \left(\alpha_{k}\right) \quad(1 \leq h \leq m) .
$$

However, it does not seem at all easy to estimate the size of the numbers $a_{h k}$ and $R_{h}$, which the applications such as those in [1] and [2,3] require.

\section{References}

[1] A. Baker, "On some diophantine inequalities involving the exponential function", Canad. J. Math. 17 (1965), 616-626.

[2] Kurt Mahler, "Zur Approximation der Exponentialfunktion und des Logarithmus. Teil I", J. reine angew. Math. 166 (1932), 118-136.

[3] Kurt Mahler, "Zur Approximation der Exponentialfunktion und des Logarithmus. Teil II", J. reine angew. Math. 166 (1932), 137-150.

[4] K. Mahler, "Perfect systems", Compositio Math. 19 (1968), 95-166.

[5] A.J. van der Poorten, "Perfect approximation of functions", Bulz. AustraZ. Math. Soc. 5 (1971), 117-126.

\footnotetext{
School of Mathematics, University of New South Wales, Kensington, New South Wales.
} 